\title{
EISCAT verification in the development of ionospheric tomography
}

\author{
I. K. Walker, J. A. T. Heaton, L. Kersley, C. N. Mitchell, S. E. Pryse, M. J. Williams \\ Department of Physics, University of Wales, Aberystwyth, SY23 3BZ, UK
}

Received: 26 February 1996/Revised: 6 May 1996/Accepted: 15 May 1996

\begin{abstract}
This paper highlights the important role played by the EISCAT radar for verification in the development of tomographic techniques to produce images of ionospheric electron density. A brief review is given of some of the stages in the application of tomographic reconstruction techniques to the ionosphere. Results are presented to illustrate the effectiveness of the method in imaging ionospheric structures at high latitudes. In addition, the results include the first tomographic image of the ionosphere for a region extending from mid-latitudes over mainland Scandinavia to high latitudes above Svalbard.
\end{abstract}

\section{Introduction}

In the past few years ionospheric tomography has become an established technique for imaging ionospheric electron density. The application of tomographic techniques to the imaging of electron density was first suggested by Austen et al. (1986). In the ionospheric application, the line integral of electron density, known as the total electron content (TEC), is measured over a large number of intersecting paths crossing a region of interest. The resulting data set is then inverted in a reconstruction algorithm to give a two-dimensional image of the electron density, usually in a height versus latitude plane. EISCAT has played a vital role in the development of ionospheric tomography by providing independent observations of electron density in a region at auroral latitudes where the horizontal structuring of the ionosphere on a scale of tens to hundreds of kilometres is well suited to imaging by the tomographic method. Pryse and Kersley (1992) reported the first independent verification of a tomographic image using electron-density measurements provided by the EISCAT radar. Whilst this represented a rudimentary initial test of the potential application of tomographic techniques to image the ionosphere, results from a later, more sophisticated experimental campaign reported by Raymund et al. (1993) produced two images of the main trough which were compared to measurements taken using the EISCAT common program CP3f. Subsequent developments have involved the use of a UK Special Program for the radar, designed specifically to take measurements of electron density for independent comparison with tomographic reconstruction images. The availability of the density measurements from EISCAT has proved highly beneficial in assessing various refinements to the algorithms used for the production of tomographic reconstructions. A brief summary of these developments is given here, together with some recent results in which the radar is used for verification of a small section of a tomographic image that extends from the polar ionosphere to mid-latitudes. It can be noted that recently measurements from EISCAT have also been presented by other workers in connection with ionospheric tomography. Markkanen et al. (1995) describe an alternative approach to the inversion problem and report two results from an experimental campaign where they were able to make comparisons between reconstructed images and simultaneous EISCAT observations above Tromsø.

\section{The experiment}

Typical geometry for a tomographic experiment is shown in Fig. 1. The radio transmissions come from satellites in the Navy Navigational Satellite System (NNSS). These satellites are in near-circular polar orbits at an altitude of about $1100 \mathrm{~km}$, transmitting phase-coherent signals on 150 and $400 \mathrm{MHz}$. The received signals can be used to estimate ionospheric TEC by means of the differential carrier phase technique. Measurements of electron content made at the chain of receiving stations are then combined to reconstruct two-dimensional images of electron density on a latitude-versus-height grid. It is important to note that a limitation of ionospheric tomography is imposed by the satellite-to-receiver geometry, where 


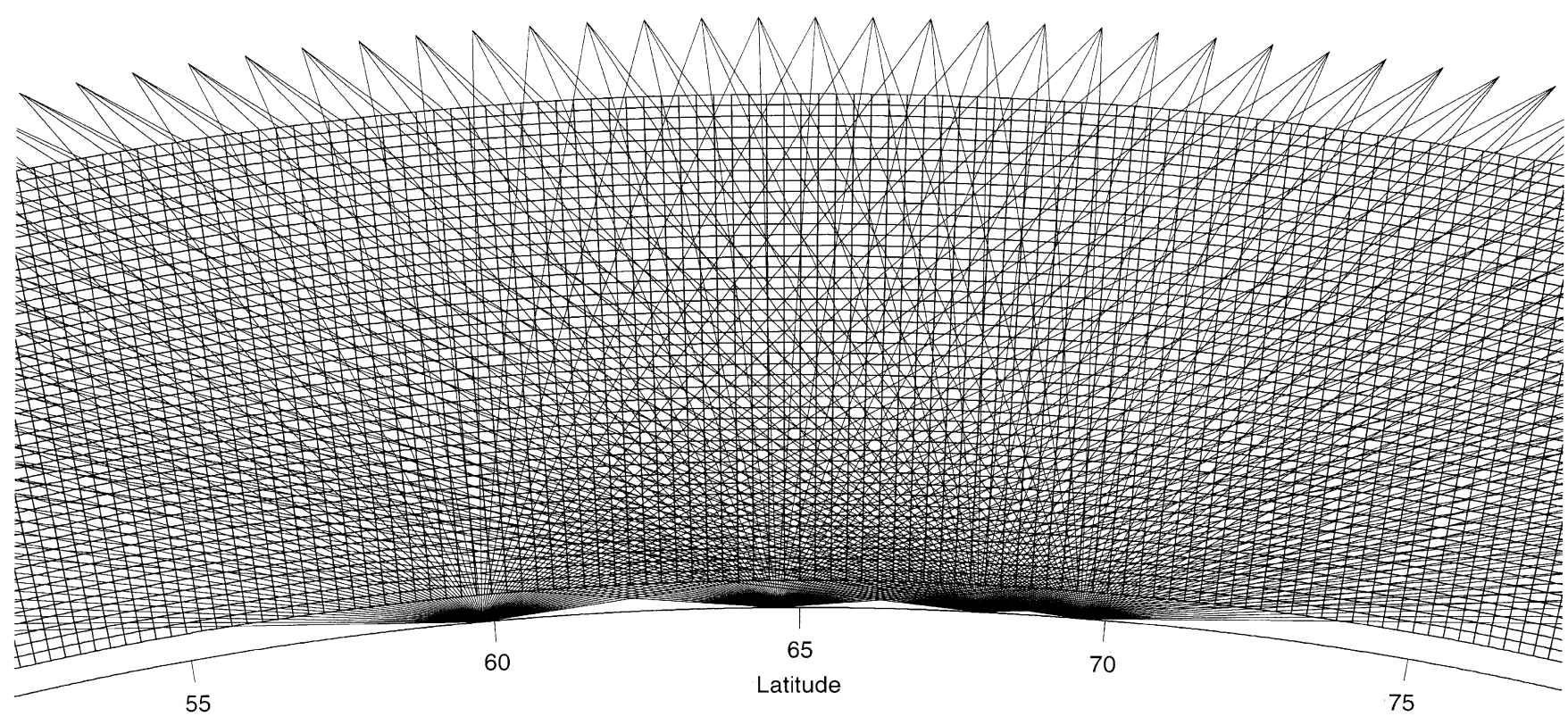

Fig. 1. Diagram showing typical satellite-to-receiver ray paths for an ionospheric tomography experiment

a lack of horizontal ray-paths through the image grid reduces the available information relating to the vertical electron-density profile. Much of the effort in the development of ionospheric tomography has been directed towards solving the problem of the vertical profile, and EISCAT has proved an invaluable tool in providing independent confirmation of the results.

Several experimental campaigns have been carried out in Scandinavia to obtain data from tomographic imaging in the vicinity of the EISCAT facility. For most of the campaigns, receiving stations were established at the four mainland locations shown on the map in Fig. 2. These sites covered some $10^{\circ}$ of latitude and were aligned approximately along a line of geographic longitude under the satellite track. More recently, use has been made of receiving sites on Svalbard. This work was intended as preparation for future studies involving both the new EISCAT Svalbard Radar (ESR) and existing EISCAT radars. Results from the first Svalbard study have been reported by Pryse et al. (1996). The success of the study led to deployment of receivers at all six locations marked on the map for the most recent campaign carried out in November 1995, the first results from which are presented here.

\section{EISCAT special program}

While the first comparison of radar and tomographic images were made using the CP3f common program, it was appreciated that this meridional scan provided too coarse a picture both temporally and spatially; a special radar program was therefore developed for this purpose. The UK-EISCAT-Special Program TOMO has been designed specifically to make measurements to determine the latitudinal and vertical structuring in electron density for use in verification of tomographic images. The

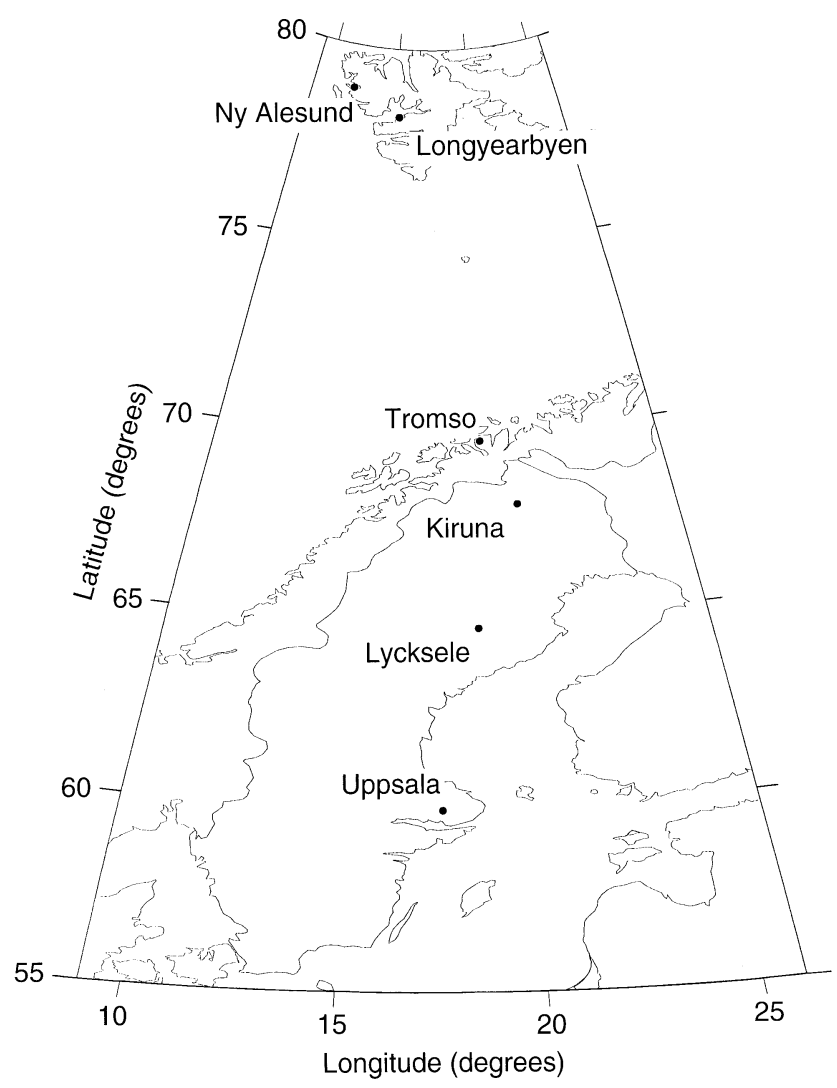

Fig. 2. Map showing the location of receiving stations used in Scandinavia

program uses the UHF radar in a meridional scan, initially with the beam pointing nearly vertical at Tromsø, with subsequent southward motion in 56 steps of $0.125^{\circ}$ $(\sim 15 \mathrm{~km})$ at $300-\mathrm{km}$ altitude. The complete scan covers some $7^{\circ}$ latitude at this height, with height resolution being retained at about $30 \pm 8 \mathrm{~km}$ through use of three radar controller programs. The cycle time for the 
repetition of the scan pattern is $20 \mathrm{~min}$, which is comparable with the horizon-to-horizon duration of a satellite pass.

\section{Results}

The problem of missing horizontal ray paths through the image region in ionospheric tomography has been addressed by supplying an estimate of the background ionosphere for initialisation of the iterative reconstruction algorithm. The vertical ionisation profile of this background is a key feature in the successful imaging of ionospheric structures. Raymund et al. (1993) described early attempts at reconstructions using a linear combination of a set of ionospheres produced by the IRI-90 model (Bilitza, 1990) to create a background ionosphere to initialise the Multiplicative Algebraic Reconstruction Technique (MART) algorithm. Whilst these first attempts showed broad agreement between horizontal structures produced using tomographic imaging and those observed using EISCAT, it was found that the ionospheric model, and hence the tomographic image, produced unrealistic topside profiles under conditions of high sunspot number. In subsequent work, Kersley et al. (1993) made use of the USU model (Daniell, 1991) which was found to give better representation of the topside ionisation distribution at high latitudes. Whilst improved agreement was found between the tomographic images and corresponding EISCAT observations, the results revealed limitations in the sole use of ionospheric models to describe the background ionosphere at high latitudes. It was noted that the models are designed to represent average conditions, which they do with varying degrees of success. However, they are less able to give an accurate description of extreme ionospheric conditions where the vertical ionisation profile can show significant departures from the average.

In an attempt to overcome these limitations, Kersley et al. (1993) incorporated ionosonde information, in the form of maximum electron density and layer peak height, to scale the ionospheric model used to initialise the MART algorithm. A range of topside profiles with peak heights and densities consistent with the ionosonde measurements was created by artificially altering the $f 10 \cdot 7$ parameter input to the IRI-90 model. A later development of this approach was reported by Heaton et al. (1995), where the topside shape was no longer model dependent but was selected from a range of possible shapes provided from a large data base of normalised topside profiles, compiled using data from the EISCAT and Malvern incoherent scatter radars. Once again the MART algorithm was used to generate the reconstuction. The success of this technique is illustrated in Fig. 3, which shows two attempts at the imaging of the main trough seen in observations from a satellite pass monitored by the chain of receivers at 1818 UT on 23 March 1992, with the relevant EISCAT observations included for comparison. It should be noted that the start and end times for monitoring of a given satellite pass differ at each receiving site, dependent upon the position in the receiving chain. For the purposes of this paper, the time used to identify a satellite pass is that at which the satellite crossed $65^{\circ} \mathrm{N}$, approximately the midpoint of the receiving chain. The image shown in Fig. 3a was produced using the IRI-90 model with ionosonde input for the initial background. Figure $3 \mathrm{~b}$ shows an image formed using the technique described by Heaton et al. (1995), while Fig. 3c displays the corresponding density measurements made using the UK EISCAT Special Program TOMO. It should be noted that the first image in this example was discussed in detail by Kersley et al. (1993), where attention was drawn to the need to calibrate correctly the TEC measurements made at each receiving site. However, the example has been included again here to illustrate the improvements made in the intervening years to the reconstruction technique.

Comparing a and $\mathrm{c}$ in Fig. 3, it can be seen that the first attempt at incorporating ionosonde information with the IRI-90 model gave a reconstruction which correctly reproduced a trough above $65^{\circ} \mathrm{N}$; however, it overestimated the densities at the layer peak and on the topside. In the case of Fig. 3b, where ionosonde information and the free range of normalised topside profiles have been used to provide the background, the position of the trough is again accurately reproduced; but now the shape of the topside is much improved when compared to the EISCAT observations (Fig. 3c). However, it can be noted that the vertical gradient on the bottomside in the image is still too sharp, so that the densities at the peak are still overestimated. The TOMO scan provides power-profile information in addition to the observations plotted in Fig. 3c which can be used to give an indication of relative densities down to about $100 \mathrm{~km}$. These show the presence of significant ionisation in the E-region that may explain the overestimation of peak densities in the reconstruction, since no account of an E-layer was taken in the initialisation of the background.

In a parallel development, attempts have been made to generate images without the use of additional ionosonde data. Under certain circumstances, for example blanketing auroral-E, ionosondes are unable to provide maximum densities and peak heights to scale the ionospheric models. The most successful approach to date involves a three-stage process, which differs significantly from the previous approaches described earlier, where a singlebackground ionosphere was used to initialise the MART algorithm. In the first stage, a range of possible background ionospheres is generated by inserting Chapman profiles, scaled using the IRI-90 model, across the image grid. This process is repeated for profiles with a range of peak heights and bottomside scale heights to create a large set of possible background ionospheres. For the second stage, a variation of the Algebraic Reconstruction Technique (ART) routine, with weighting along the ray paths, is used to generate a reconstruction for each of the background ionospheres that have been created. By means of a least-squares test on the resultant reconstructions, comparing the relative TEC measurements made at the receiving stations with those found for the same paths through the reconstructed images, it is possible to identify initial best estimates of peak height and peak density for the image. In the third stage of the process, the image is 

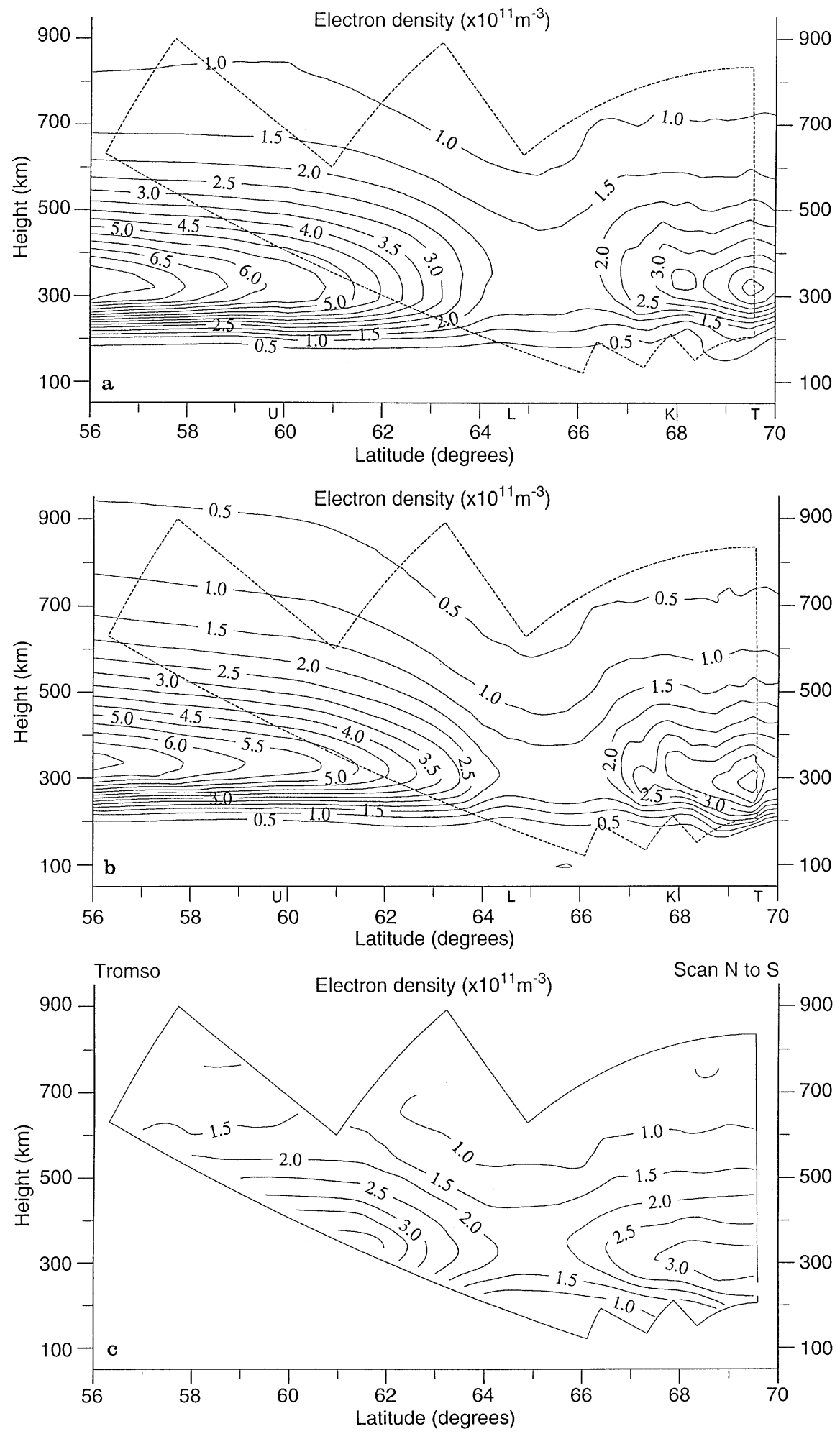

Fig. 3. a Tomographic image of electron density for a satellite pass at 1818 UT on 23 March 1992 with background ionosphere based on IRI-90 model with ionosonde input (after Kersley et al., 1993); b tomographic image of electron density for the same pass background ionosphere based on IRI-90 model incorporating ionosonde input, with topside shape selected from a large data base of normalised topside profiles provided by the Malvern and EISCAT incoherent scatter radars; c electron densities measured by EISCAT using SP-UK-TOMO between 1810 and 1829 UT on 23 March 1992

fine tuned by making further reconstructions based on backgrounds created using these best estimates of peak height and density in conjunction with the varying topside profiles described by Heaton et al. (1995) and standard Chapman bottomside profiles. Much smaller variations in the peak height and peak densities are tolerated in this final stage of the process.
An illustration of the success of this approach can be seen by comparing Fig. 4a, a reconstruction made using only the USU model to initialise the background for the reconstruction, with Fig. 4b, the image produced using the new technique. Figure $4 \mathrm{c}$ shows the relevant EISCAT observations made using SP-UK-TOMO. In this example, an unusually high peak height at around $475 \mathrm{~km}$ 

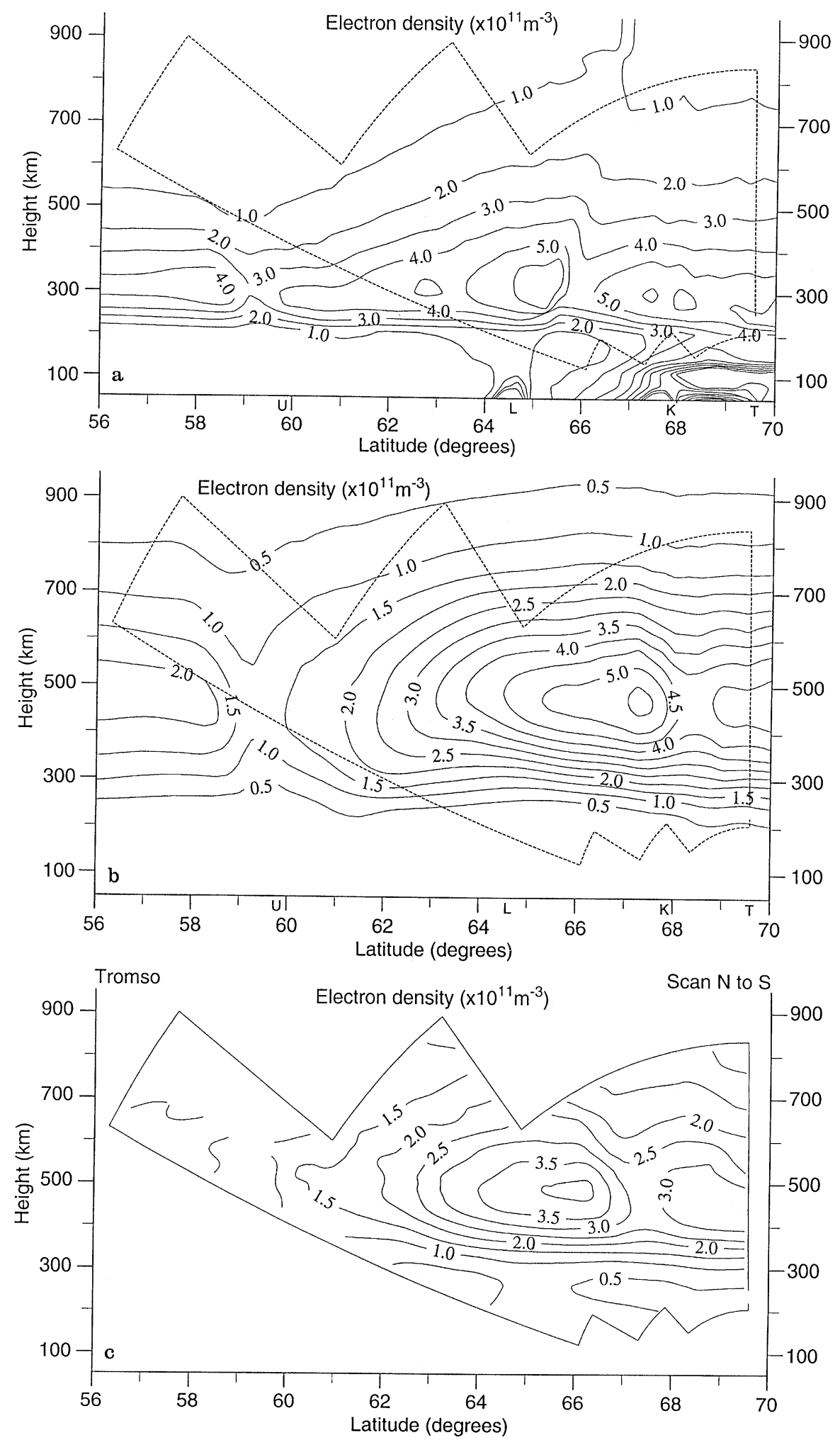

Fig. 4. a Tomographic image of electron density for a satellite pass at 2058 UT on 23 March 1992 with background ionosphere based on the USU model (taken from Kersley et al., 1993); b tomographic image of electron density for the same pass with background ionosphere based on latest technique which requires no additional a priori information (see text); c electron densities measured by EISCAT using SP-UK-TOMO between 2050 and 2109 UT on 23 March 1992

was observed by EISCAT. Figure 4a shows that the initial attempt at reconstruction using only the USU model completely failed to reproduce the correct peak height. There appears to be a broad match in the tomographic image and EISCAT plot between the locations of the trough to the south and the density enhancements further north. However, the peak densities yielded by the recon- struction are significantly higher than those seen using EISCAT. In contrast, the image produced by the new method shown in Fig. 4b has accurately replicated the height of the F-layer peak. This represents a significant step forward in the development of ionospheric tomographic reconstruction techniques, without recourse to additional data sources for use in the reconstruction. The 


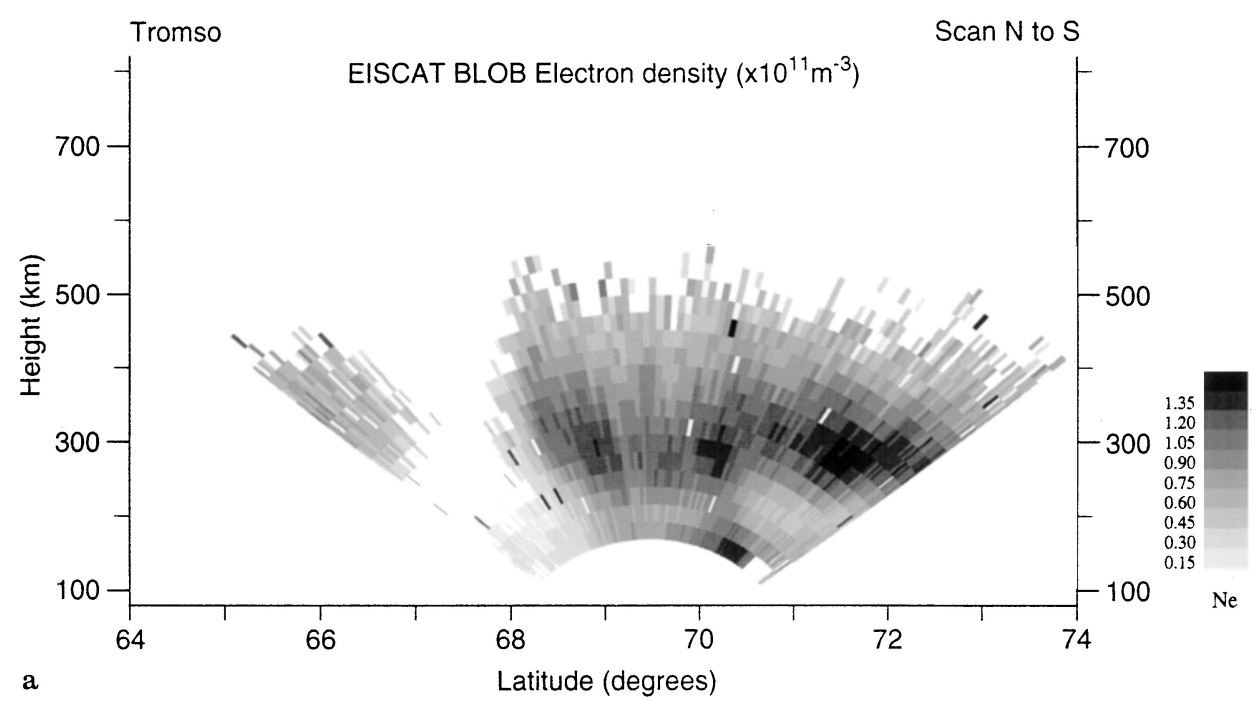

a

Latitude (degrees)

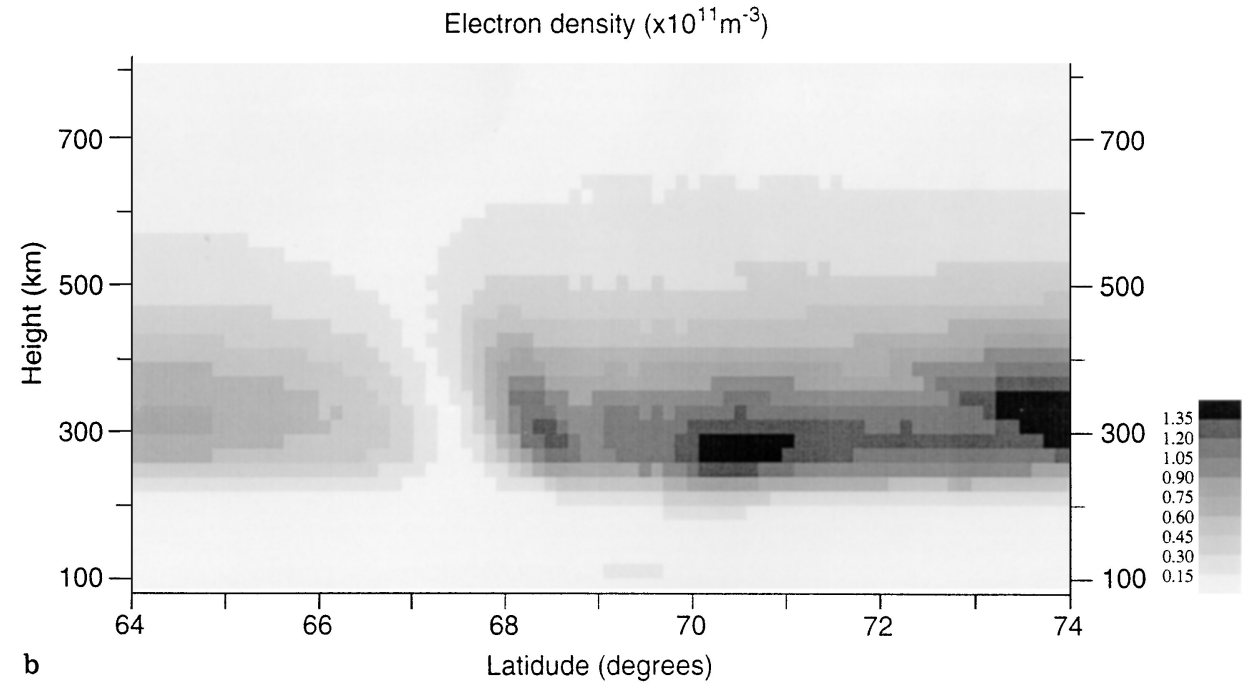

Fig. 5. a Electron-density measurements made by EISCAT during a scan of SP-UK-BLOB between 2102 and 2128 UT on 15 October 1993; b tomographic image of electron density for a satellite pass at 2136 UT on 15 October 1993. (after colour plots presented by Mitchell et al., 1995)

locations of the main features in the horizontal structure of the image match reasonably well with the EISCAT observations, but there are still discrepancies between the magnitudes of the densities, with the reconstructed image again showing higher densities than those observed by EISCAT. Here again the power-profile data showed the presence of significant ionisation in the E-region which had not been taken into account with the initialisation of the background. This may well account for the observed discrepancies.

A second UK EISCAT Special Program (SP-UKBLOB) has also been used in the verification of tomographic images. The primary aim of this routine is the study of density structures in the auroral $F$ region with transverse scales of the order of tens of kilometres. Full details of BLOB can be found in Pryse et al. (1996). Figure 5a shows the electron-density measurements made by EISCAT during a scan of the BLOB program between 2102 and 2128 UT on 15 October 1993. These data are taken from a 7-h run of the experiment during very quiet geomagnetic conditions, where $K p$ was about $0+$. A trough can be seen at the southern end of the scan. The densities in the trough minimum are so low that the signal-to-noise ratio measured by the radar was below the threshold for reliable analysis. A steep gradient in electron density forms the poleward wall of the trough, where a region of density enhancement is elongated along the direction of the geomagnetic field. To the north, additional structuring is apparent, with indications of structure at a lower peak height around $70^{\circ} \mathrm{N}$ and some evidence of auroral-E above this latitude.

The tomographic image from a satellite pass about the time of these observations is shown in Fig. 5b, the satellite crossing $65^{\circ} \mathrm{N}$ at approximately $2136 \mathrm{UT}$. Excellent agreement can be seen between the image and EISCAT observations, particularly at the poleward wall of the trough with the field-aligned enhancement in density. Furthermore, there is also indication of structure at a lower peak height around $70^{\circ} \mathrm{N}$. The densities in the trough minimum are determined by the tomographic method, showing clearly the narrow field-aligned structure of the trough in this instance. This probably represents the best experimental tomographic reconstruction to date. The pixel size used (approximately $15 \times 20 \mathrm{~km}$ at F-region altitudes) is comparable to that of the radar observations and 


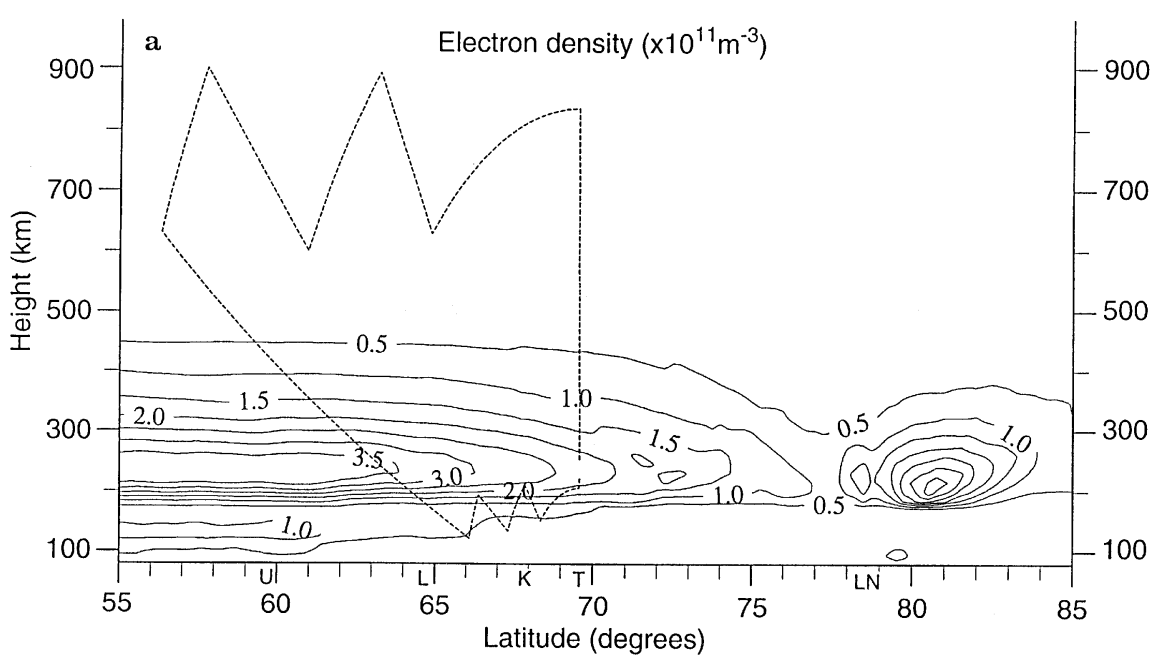

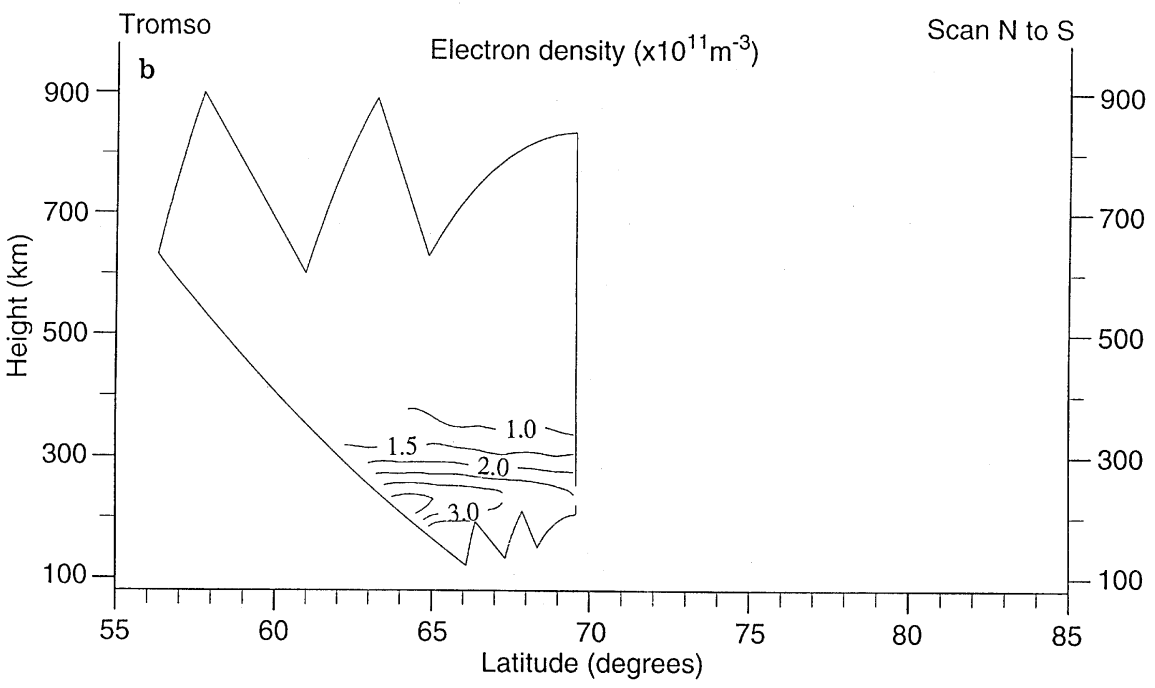

Fig. 6. a Tomographic image of electron density for a satellite pass at 1122 UT on 23 November 1995; b electron densities measured by EISCAT using SP-FI-TOMO between 1116 and 1134 UT on the 23 November 1995 is approaching the diffraction limit imposed by phase scintillation that controls the ultimate resolution of the method. Mitchell et al. (1995) have presented power-profile data from the radar for this time that give relative electron densities at lower altitudes. These confirm the presence of an auroral-E layer beneath structured enhancements poleward of the trough. By reducing the data separation interval and thereby increasing the number of ray paths in the image grid, these authors were able to begin to form the increased densities to the E-layer in the reconstruction.

In an experimental project in November 1995, all six receiving stations marked on the map shown in Fig. 2 were used. This represented the first attempt at imaging the ionosphere over a region extending from mid-latitudes above mainland Scandinavia to polar latitudes over Svalbard. Figure 6a shows a reconstruction generated using measurements made at all of the sites and covering some $30^{\circ}$ of latitude. For comparison, the relevant EISCAT data obtained from a run of the Russian/Finnish Special Program TOMO are shown in Fig. 6b. It can be noted that this special program is a derivative from the UK TOMO program, with scanning taking place in the geomagnetic meridian rather than the geographic plane.
The method employed to generate the reconstruction includes the use of a complete bottomside profile from the Tromsø dynasonde to scale the IRI-90 model that is used to form the background for initialisation of the reconstruction algorithm.

The main features that can be seen in the reconstructed image from a satellite pass at about $1120 \mathrm{UT}$ are the trough-like depletion just above $77^{\circ} \mathrm{N}$, with evidence of density structuring polewards of this region and a relatively smooth increase in density to the south. Comparison in the viewing area of EISCAT shows that the equatorward gradient has been well replicated in the tomographic image, with both the peak height and densities exhibiting good agreement. This agreement, found over a segment of the image, places confidence in the authenticity of the electron densities revealed by the tomographic method over the wider region. Of particular interest in the reconstructed image are the features seen above the receivers on Svalbard (Longyearbyen $78 \cdot 2^{\circ} \mathrm{N}, \mathrm{Ny}$ Alesund $78 \cdot 9^{\circ} \mathrm{N}$ ). It is well established that density structuring at high latitudes is dominated by the convection flow driven by the solar wind. For observations made at receiving sites on Svalbard, the ionospheric satellite-to-receiver ray-path 


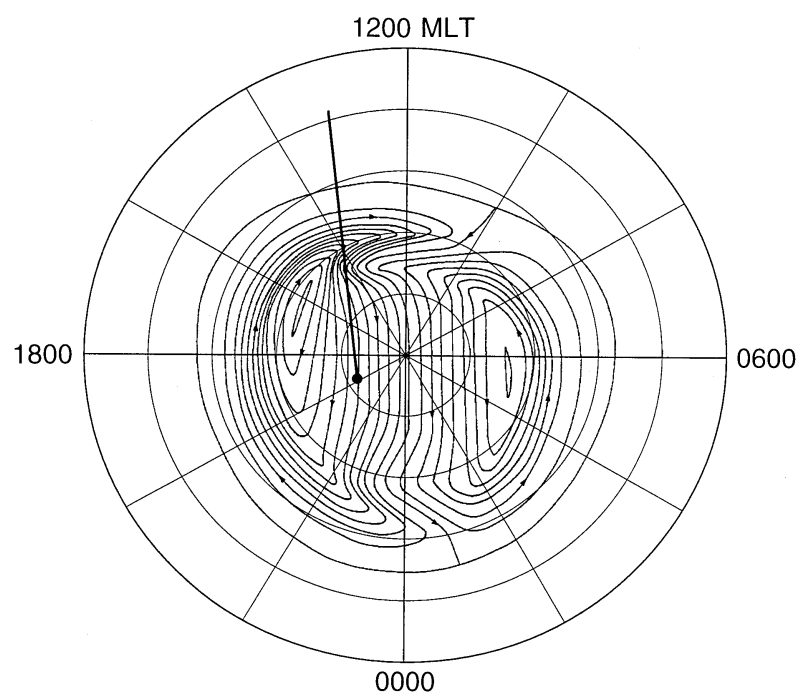

Fig. 7. Geometry of the ray-path intersection at a height of $300 \mathrm{~km}$ for the satellite pass at 1122 UT on 23 November 1995 on a geomagnetic latitude $\left(90^{\circ}\right.$ to $\left.40^{\circ}\right)$ versus MLT grid. The Heppner and Maynard Model A two-cell convection pattern for $B_{\mathrm{z}}$ and $B_{\mathrm{y}}$ negative has been superimposed

intersection can encounter markedly different regions in the convection pattern at different times during the pass. Figure 7 shows the relevant ionospheric intersection track for the observations presented as a function of magnetic local time and magnetic latitude. Superimposed on the plot is a schematic representation of a simple two-cell convection pattern for $B_{z}$ negative taken from Heppner and Maynard (1987). The satellite was moving from higher to lower latitudes, and it can be seen that initially the track might have encountered plasma moving in the antisunward flow across the polar cap and then could have gone on to pass through the throat region for this flow. As the satellite moved further south, the track would intersect plasma corotating in winter darkness at high latitudes and finally the sunlit near-noon ionosphere at mid-latitudes. With this in mind, the structures can be interpreted. The enhancement seen above $81^{\circ} \mathrm{N}$ geographic in the tomographic image is likely to be a section elongated in the convective flow through a so-called 'patch', starting its journey across the polar cap. Similarly, the smaller enhancement seen above $78 \cdot 5^{\circ} \mathrm{N}$, and possibly field-aligned, is a transverse section in the postmagnetic noon region of the tongue of ionisation, shown by models to feed into the polar cap. It can also be noted that this enhancement might be linked to particle precipitation in the cusp region. Indeed, the reconstruction has formed a small region of E-layer ionisation near $79^{\circ} \mathrm{N}$ that may be further evidence of particle precipitation in this region.

The trough, with minimum between $77^{\circ} \mathrm{N}$ and $78^{\circ} \mathrm{N}$, occurs on flux tubes that have long been in winter darkness, either in the return flow of the afternoon cell or co-rotating at high latitude. To the south there is the latitudinal gradient of the sunlit daytime ionosphere. It can be noted that some of the apparent structure at the northern edge of this gradient arises from artefacts in the reconstructed image. It is well understood that spurious features or 'wings' can be produced in an image in regions where ray-path coverage is lost from individual stations. The wide gap between the stations on Svalbard and at Tromsø results in such features that can be seen as an artificial elongation of the $1 \times 10^{11} \mathrm{el} \mathrm{m}^{-3}$ contour between $75^{\circ} \mathrm{N}$ and $77^{\circ} \mathrm{N}$. This effect is also apparent in the elongation of the northern edge of the 'patch' feature at latitudes greater than $81^{\circ} \mathrm{N}$. However, in spite of these limitations the reconstruction presented in Fig. 6a demonstrates the potential of the tomographic method for the imaging of large sections of ionospheric electron density.

\section{Conclusions}

The EISCAT radar has played an important role in the development of the application of tomographic reconstruction techniques to the imaging of the ionospheric electron density. The radar has provided independent measurements of the electron concentration that have been used to assess the performance of various algorithms developed for the inversion of the experimental observations of TEC. Without such independent verification the development of ionospheric tomography is restricted to consideration of modelling studies that are often less than realistic representations of the actual situation encountered in experimental applications. The experimental results given here illustrate some of the progress made in ionospheric tomography using EISCAT comparisons. It can be noted that comparisons have been published relating tomographic images to observations made using the Millstone Hill radar (Foster et al., 1994; Pakula et al., 1995). Recently, Markkanen et al. (1995) have reported a new approach to tomographic inversion in which observations by the EISCAT radar are not used for verification but form an input to the reconstruction process itself. The results presented, particularly those at high latitudes, demonstrate the potential of the tomography method to provide information on ionospheric electron densities over wide spatial regions from observations at a few ground-based locations.

Acknowledgements. This work has been supported by the UK Particle-Physics and Astronomy Research Council. The assistance of the director and staff of the EISCAT Scientific Association, the staff of the Norsk Polarinstitutt at Ny Ålesund and the director and staff of the Swedish Institute of Space Physics is gratefully acknowledged. In addition the authors would like to thank Professor Evgeny Tereshchenko of the Polar Geophysical Institute in Mumansk, Russia and Dr Tuomo Nygrén of the University of Oulu, Finland for provision of data from EISCAT special program time during the November 1995 campaign.

Topical Editor D. Alcaydé thanks E. J. Fremouw and another referee for their help in evaluating this paper.

\section{References}

Austen, J. R., S. J. Franke, and C. H. Liu, Application of computerized tomography techniques to ionospheric research, in Radio Beacon Contributions to the Study of Ionization and Dynamics of the Ionosphere and to Corrections to Geodesy and Technical Workshop, ed. A. Tauriainen, Ouluensis Universitas, Oulu, Finland, Beacon Satellite Symposium, 1986. 
Bilitza, D., International Reference Ionosphere 1990, National Space Science Data Center/World Data Center A for Rockets and Satellites 90-22, 1990.

Daniell, R. E., Parameterized Real-Time Ionospheric Specification Model: PRISM Version 1.0, Phillips Laboratory Technical Report, PL-TR-91-2299, Hanscom AFB, MA 01731, USA, 1991.

Foster, J. C., M. J. Buonsanto, J. M. Holt, J. A. Klobuchar, P. Fougere, W. Pakula, T. D. Raymund, V. E. Kunitsyn, E. S. Andreeva, E. D. Tereshchenko, and B. Z. Khudukon, RussianAmerican Tomography Experiment, Int. J. Image Syst. Technol., 5, 148-159, 1994.

Heaton, J. A. T., S. E. Pryse, and L. Kersley, Improved background representation, ionosonde input and independent verification in experimental ionospheric tomography, Ann. Geophysicae, 13, 1297-1302, 1995.

Heppner, J. P., and N. C. Maynard, Empirical High-Latitude Electric Field Models, J. Geophys. Res., 89, 44467-4489, 1987.

Kersley, L., J. A. T. Heaton, S. E. Pryse, and T. D. Raymund, Experimental ionospheric tomography with ionosonde input and EISCAT verification, Ann. Geophysicae, 11, 1064-1074, 1993.
Markkanen, M., M. Lehtinen, T. Nygrén, J. Pirttilä, P. Henelius, E. Vilenius, E. D. Tereshchenko, and B. Z. Khudukon, Bayesian approach to satellite radiotomography with applications in the Scandinavian sector, Ann. Geophysicae, 13, 1277-1287, 1995.

Mitchell, C. N., D. G. Jones, L. Kersley, S. E. Pryse, and I. K. Walker, Imaging of field-aligned structures in the auroral ionosphere, Ann. Geophysicae, 13, 1311-1319, 1995.

Pakula, W. A., P. F. Fougere, J. A. Klobuchar, H. J. Kuenzler, M. J. Buonsanto, J. M. Roth, J. C. Foster, and R. E. Sheehan, Tomographic reconstruction of the ionosphere over North America with comparisons to ground-based radar, Radio Sci., 30, 89-103, 1995.

Pryse, S. E., and L. Kersley, A preliminary experimental test of ionospheric tomography, J. Atmos. Terr. Phys., 54, 1007-1012, 1992.

Pryse, S. E., L. Kersley, and I. K. Walker, Blobs and irregularities in the auroral ionosphere, J. Atmos. Terr. Phys., 58, 205-215, 1996.

Raymund, T. D., S. E. Pryse, L. Kersley, and J. A. T. Heaton, Tomographic reconstruction of ionospheric electron density with European incoherent scatter radar verification, Radio Sci., 28, 811-817, 1993. 